\title{
Diferenciação de cervejas usando fosforimetria
}

\author{
Carlos Eduardo Cardoso
}

Universidade Severino Sombra

\section{Cardoso@uss.br}

\begin{abstract}
Resumo Cerveja é uma mistura complexa que consiste principalmente de água e etanol, com 0,5\% de sólidos dissolvidos. A caracterização de amostras complexas nestes casos, normalmente emprega técnicas analíticas baseadas na separação destes componentes. Alternativamente, o uso de métodos espectroscópicos vem sendo reconhecido como método rápido e sensível, com aplicabilidade para uma grande variedade de produtos. Assim, o presente trabalho teve por objetivo aplicar a fosforimetria em substrato sólido na temperatura ambiente para a caracterização de amostras de dois tipos de cervejas produzidas no mercado nacional através do efeito externo do átomo pesado e do efeito de um modificador de superfície na indução da fosforescência. Como resultado, uma metodologia para caracterização de amostras de cervejas foi desenvolvida. Parâmetros experimentais e instrumentais foram otimizados com o intuito de minimizar a interferência entre os processos radiativos. Isso foi conseguido pela combinação do uso de surfactante SDS 0,07 $\mathrm{mol} \mathrm{L}^{-1}$ e de átomo pesado, KI 1,0 mol $L^{-1}$ e também pela utilização de tempos adequados de abertura do detector e tempo de retardo.
\end{abstract}

Palavras-Chave: Fosforimetria, Cervejas, Efeito externo do átomo pesado, modificador de superficie

\begin{abstract}
Beer is a complex mixture that mainly consists of water and ethanol, with 0,5\% of dissolved solids. The characterization of complex samples normally use analytical techniques in these components separation. Alternatively, the use of spectroscopic methods comes being recognized as fast and sensible method, with applicability for a great variety of products. Thus, the present work had for objective apply the solid surface room temperature phosphorimetry for two beers characterization through the heavy atom external effect and a surface modifier effect. As result, a methodology for beer samples characterization was developed. Experimental and instrumental parameters had been optimized to minimize the radioactive processes interference. This was obtained by the combination of the 0,07 $S D S$ mol L $L^{-1}$ and KI 1,0 mol L $L^{-1}$ se and adequate times of detector opening and retardation time.
\end{abstract}

Keywords: Phosphorimetry, Beer, Heavy-atom induced phosphorescence, surface modifier

\section{Introdução}

Cerveja é uma mistura complexa que consiste principalmente de água e etanol, com $0,5 \%$ de sólidos dissolvidos [1]. A qualidade, a homogeneidade e a estabilidade são importantes aspectos que preocupam as indústrias de alimentos e bebidas em geral. A caracterização de amos- tras complexas nestes casos, normalmente emprega técnicas analíticas baseadas na separação dos componentes das amostras.

A análise de metais por espectrometria atômica de chama assim como espectros de absorvância molecular foram usados por Bellido-Mila e colaboradores [2] para diferenciar e agrupar diferentes tipos de cerveja. No en- 


\section{Revista Eletrônica TECCEN - ano I - volume 2 - Out-Dez de 2008 ISSN 1984-0993}

tanto, um pré-tratamento da amostra foi necessário para a análise dos metais.

O uso de métodos espectroscópicos na análise de alimentos vem sendo reconhecido como método rápido e sensível, com aplicabilidade para uma grande variedade de produtos. $\mathrm{Na}$ análise de cerveja, existem diversos artigos que usam a fluorimetria como método de detecção de vários compostos. Sikorska e colaboradores1 usaram a espectroscopia de fluorescência para monitorar possíveis alterações do produto ao longo do tempo de armazenamento. Este trabalho indica a correspondência das bandas observadas na cerveja com as bandas observadas para a riboflavina. Em relação a fosforimetria aplicada a análise de cervejas Cañabate-Diaz e colaboradores [3] relatam o seu uso na determinação de triptamina em amostras de cerveja.

Assim, o presente trabalho teve por objetivo aplicar a fosforimetria em substrato sólido na temperatura ambiente para a caracterização de amostras de dois tipos de cervejas produzidas no mercado nacional através do efeito externo do átomo pesado e do efeito de um modificador de superfície na indução da fosforescência dos analitos. Após obtidas as melhores condições para a obtenção do sinal fosforescente das amostras, foram traçados mapas de contorno e superfícies de projeção (espectros 3D) usando o software FL WinLab/3D Viewer da PerkinElmer.

\section{Metodologia}

Dos vários fenômenos que se seguem à exposição de uma molécula orgânica insaturada à radiação eletromagnética, são de especial interesse para este trabalho os fenômenos fotoluminescentes, mais especificamente a fosforescência.

A fosforimetria em superfície sólida na temperatura ambiente (FSSTA) é um procedimento experimental simples, rápido e de baixo custo. No papel, o analito é imobilizado por dois mecanismos. Primeiro pela interação (ligações de hidrogênio) entre os grupos $-\mathrm{OH}$ da celulose com os elétrons $\pi$ ou outros grupos eletronegativos da molécula do analito. A segunda interação importante é o encapsulamento físico das moléculas do analito nos poros do substrato de papel. Esses dois mecanismos dão a rigidez necessária para permitir o aumento da eficiência quântica fosforescente de analitos de várias classes químicas. As duas principais desvantagens do substrato de papel são o seu relativo alto sinal de fundo e a necessidade do uso de fluxo de gás inerte direcionada para amostra, cujo objetivo é a eliminação do oxigênio do ambiente.
O papel (celulose) é um substrato bastante versátil, facilmente disponível e fácil de se trabalhar quando comparados com a sílica, polímeros, açúcares e outros que têm sido utilizados. Assim, o papel tem sido o substrato de preferência em muitos trabalhos com fosforimetria na temperatura ambiente [4]. Levando-se em consideração o fato de que na FSSTA os substratos de papel não são compostos puramente de celulose, mas também de hemicelulose, lignina e até de metais de transição, diversos autores têm testado vários tipos de papéis e procedimentos alternativos de tratamento dos mesmos para redução do sinal de fundo [4], obtendo-se redução de até $97 \%$.

O aumento da eficiência da emissão fosforescente pode ser promovido pelo efeito do átomo pesado (HAE). Alguns autores [5] têm explicado este efeito pela teoria do acoplamento spin-orbital, onde o movimento orbital dos elétrons gera um campo magnético que desvia o momento angular do spin dos elétrons do analito, dando um caráter triplete $(\mathrm{T})$ à molécula que se encontra no estado singlete $(\mathrm{S})$, o que diminui o caráter proibitivo da transição entre estados de diferentes multiplicidades. Desta forma, o átomo pesado favorece este acoplamento, aumentando assim a população do estado $T_{1}$ responsável pela emissão fosforescente. Da especificidade da ação do átomo pesado (aumento ou redução do sinal fosforescente e/ou afinamento da banda de emissão) sobre o sinal da molécula do analito, derivou-se a técnica conhecida por Perturbação Seletiva do Átomo Pesado Externo (SPHA). A técnica consiste, basicamente, no aumento seletivo que um átomo pesado pode provocar no sinal fosforescente de um componente específico, aspecto este interessante do ponto de vista da determinação de compostos de interesse em misturas complexas sem a necessidade de etapas prévias de separação.

Além da potencial indução ou amplificação do sinal fosforescente (efeito externo de átomo pesado), a presença destes íons pode também aumentar a velocidade dos processos de desativação do estado excitado sem emissão de radiação. Neste caso, o sinal fosforescente não será observado.

Em muitos casos, a interação entre o íon de átomo pesado e a molécula de analito pode ser melhorada com o uso de modificadores de superfície (agentes surfactantes) que aumentam a proximidade entre as duas espécies químicas envolvidas no processo e, consequentemente melhoram a interação. O dodecil sulfato de sódio (SDS) é geralmente o agente surfactante mais utilizado sendo colocado no substrato de papel previamente à colocação da solução de átomo pesado e do analito $(5 \mu \mathrm{L}$ em todos os casos).

Uma alternativa para maior discriminação das informações contidas nos espectros, normalmente traçados em duas dimensões (intensidade de sinal vs. comprimento de 


\section{Revista Eletrônica TECCEN - ano I - volume 2 - Out-Dez de 2008 ISSN 1984-0993}

onda), tem sido o tratamento desse conjunto de valores que, após normalização em uma matriz de dados adequada, pode ser convertida em uma imagem em três dimensões, mais rica em detalhes. A visualização da imagem pode se dar pela projeção da superfície, na qual podem ser alterados os ângulos de projeção e rotação (espectros 3D) ou ainda, através de um mapa de contorno, plotado em duas dimensões, no qual são unidos os pontos de intensidades iguais. Adicionalmente, um programa computacional adequado pode gerar cortes verticais e horizontais dos espectros $3 \mathrm{D}$, detalhando apenas uma determinada área de interesse.

\section{Experimental}

\subsection{Instrumental}

O trabalho foi desenvolvido em um espectrofluorímetro (LS 55 Perkin-Elmer) com fonte de excitação pulsada do tipo descarga de xenônio, e detector do tipo fotomultiplicador com resposta espetral S5. O instrumento é composto por dois monocromadores independentes, com redes de difração de 1200 ranhuras $\mathrm{nm}^{-1}$. As amostras e os brancos foram acomodadas em suporte para substrato sólido, ao qual foi adaptado um sistema para purga com nitrogênio.

\subsection{Reagentes}

Todos os reagentes empregados possuiam grau analítico. A água utilizada apresentou resistividade de cerca de 18,2 $\mathrm{M} \Omega \mathrm{cm}$, tendo sido deionizada em um sistema Milli-Q (Milli-Q Plus). As soluções de átomos pesados foram preparadas a partir de seus sais (nitrato de tálio, nitrato de chumbo e iodeto de potássio, obtidos da Merk Brasil).

\subsection{Procedimentos}

\subsubsection{Preparação do substrato sólido}

O papel de filtro Whatmam $\mathrm{n}^{\circ} 42$ foi lavado com água quente para retirada de impurezas durante duas horas em extrator Soxhlet. Após secagem, foram cortados discos de $1,5 \mathrm{~cm}$ de diâmetro, os quais foram expostos a radição UV por duas horas. Para a irradiação dos discos, foi utilizado um reator fotoquímico construído para este fim no próprio laboratório, cujo sistema é constituído por seis lâmpadas de emissão máxima $\left(\lambda_{\max }\right)$ a 300 nm (lâmpada para esterilização de vapor de mercúrio, $6 \mathrm{~W}$ cada, Microbiológica, Rio de Janeiro, Brasil).

\subsubsection{Procedimento para medição da fosforescência}

Inicialmente, duas marcas de cervejas do tipo Premium, foram selecionadas como amostras de interesse em virtude de serem comumente encontradas no comércio local e de representarem produtos largamente utilizados pelos consumidores deste tipo de bebida.

A fosforescência das amostras foi avaliada em papel de filtro Whatman $n^{\circ} 42$ previamente tratado para redução do sinal de fundo, que funcionou como substrato sólido. A desoxigenação do ambiente da amostra e do aparato de suporte do substrato foi feita por insuflação de gás nitrogênio isento de oxigênio e de umidade.

Para cada tipo de cerveja, foram realizados os seguintes estudos (os parâmetros experimentais e instrumentais utilizados podem ser vistos na Tabela 1):

a. Estudo das características fosforescentes em substrato de celulose;

b. Estudo do efeito de íons de átomos pesados $\left(\mathrm{Tl}^{+} 0,1 \mathrm{~mol} \mathrm{~L}^{-1}, \mathrm{I}^{-} 1,0 \mathrm{~mol} \mathrm{~L}{ }^{-1}, \mathrm{~Pb}^{+2} 0,25 \mathrm{~mol} \mathrm{~L}^{-1}\right)$ no sinal fosforescente;

c. Estudo do efeito de modificador de superfície (SDS 0,07 mol L'-1) no sinal fosforescente das amostras de interesse. 


\begin{tabular}{|c|c|c|}
\hline & $\begin{array}{l}\text { Condições } \\
\text { padrão do LS- } \\
55\end{array}$ & $\begin{array}{l}\text { Condições utili- } \\
\text { zadas neste } \\
\text { trabalho }\end{array}$ \\
\hline$\lambda_{\text {excitação }} / \lambda_{\text {emissão }}$ & -- & $275 \mathrm{~nm} \mathrm{/} 475 \mathrm{~nm}$ \\
\hline $\begin{array}{l}\text { Fendas (entrada } \\
\text { e saída) }\end{array}$ & $10 \mathrm{~nm}$ & $10 \mathrm{~nm}$ \\
\hline $\begin{array}{l}\text { Velocidade de } \\
\text { varredura }\end{array}$ & $1200 \mathrm{~nm} \mathrm{~s}^{-1}$ & $600 \mathrm{~nm} \mathrm{~s}^{-1}$ \\
\hline $\begin{array}{l}\text { Tempo de aber- } \\
\text { tura do detec- } \\
\text { tor (gate) }\end{array}$ & $1 \mathrm{~ms}$ & $9 \mathrm{~ms}$ \\
\hline $\begin{array}{l}\text { Tempo de re- } \\
\text { tardo (delay) }\end{array}$ & $1 \mathrm{~ms}$ & $0,1 \mathrm{~ms}$ \\
\hline Flash & $16 \mathrm{~ms}$ & $16 \mathrm{~ms}$ \\
\hline Átomo pesado & -- & $\begin{array}{l}0,1 ; 0,5 \text { e } 1,0 \\
\mathrm{~mol}^{-1}\end{array}$ \\
\hline Surfactante & -- & $\begin{array}{lll}\operatorname{SDS} & 0,07 & \mathrm{~mol}\end{array}$ \\
\hline
\end{tabular}

Tabela 1: Parâmetros experimentais e instrumentais

a as condições segundo Carretero e colaboradores referem-se a fosforescência em solução na temperatura ambiente da molécula de triptamina presente em amostra de cerveja sem álcool.

Encontradas as condições para indução e maximização do sinal fosforescente (possivelmente devido a presença de riboflavina e de triptamina nas cervejas), foi traçado o espectro de fosforescência total de cada amostra. Para tanto, foram feitas 13 varreduras excitando-se a partir de $210 \mathrm{~nm}$ e com sucessivos incrementos de $10 \mathrm{~nm}$. Os espectros de emissão foram coletados na faixa compreendida entre $350 \mathrm{~nm}$ e $650 \mathrm{~nm}$.

\section{Resultados}

A escolha do substrato para indução de fosforescência é parte crucial das técnicas fosforimétricas feitas na temperatura ambiente, já que a imobilização do analito permite que a molécula, ao absorver energia radiante de excitação, possa passar para o estado excitado tripleto, para então desativar-se para o estado fundamental (fosforescência). A escolha adequada do substrato de papel e a otimização do procedimento para redução do seu sinal fundo (lavagem e tratamento fotoquímico) permitiram, previamente a esta proposta, reduzir em $75 \%$ o tempo do processo de preparo e tratamento desses substratos descrito na literatura, com um decréscimo de pelo menos $90 \%$ do sinal de fundo original.

Após a escolha do substrato, tornou-se necessário otimizar (procedimento univariado) as condições experi- mentais e instrumentais visando à obtenção e a maximização dos sinais fosforescentes dos analitos na temperatura ambiente. Trabalhos já descritos anteriormente [3], relatam a fluorescência da riboflavina e a fosforescência em solução na temperatura ambiente da triptamina [4], compostos sempre presentes em cervejas.

As características fosforescentes das duas amostras foram previamente estudadas usando diferentes condições experimentais, visando identificar aquelas que forneceriam melhor desempenho em termos de resolução espectral e intensidade de sinal fosforescente. Assim, nessa etapa, o sinal de cada amostra de cerveja foi estudado no $\mathrm{pH}$ natural da amostra (cerca de 4,5) em função da presença ou não de átomo pesado $\left(\mathrm{I}^{-}, \mathrm{Tl}^{+}, \mathrm{Pb}^{2+)}\right.$ e de SDS.

Conforme os estudos demonstraram (Figura 1), o efeito do íon do metal de átomo pesado foi fundamental na indução a amplificação de fosforescência dos analitos, já que este atua aumentando a velocidade do cruzamento entre os estados excitados de diferentes multiplicidades, incrementando, assim, a população no estado excitado tripleto. Para tal estudo, alíquotas de $5 \mu \mathrm{L}$ da amostra em estudo foram depositadas em substratos de celulose nos quais foram previamente aplicada soluções de sais dos átomos pesados apropriados $\left(\mathrm{KI} 1 \mathrm{~mol} \mathrm{~L}^{-1}, \mathrm{TINO}_{3} 0,1\right.$ $\mathrm{mol} \mathrm{L}^{-1}$, e $\left.\mathrm{Pb}\left(\mathrm{NO}_{3}\right)_{2} 0,5 \mathrm{~mol} \mathrm{~L}^{-1}\right)$.

Modificadores de superfície, principalmente surfactantes, têm sido utilizados para melhorar as interações entre o analito, o íon de átomo pesado e o substrato de celulose [3,6]. O surfactante pode melhorar o contato entre o íon de átomo pesado e o analito, amplificando o efeito externo que ocasiona a mudança de multiplicidade do estado excitado. Além disso, o surfactante pode melhorar a rigidez do analito no substrato e impedir que o mesmo se infiltre por demais nas porosidades do papel, ficando indisponível para ser interrogado pela radiação de excitação. $\mathrm{O}$ efeito causado pelo surfactante tem se mostrado bastante dependente de sua quantidade no local onde o analito é aplicado, visto que usando uma grande quantidade do mesmo, pode haver um decréscimo de sinal fosforescente por causa da dificuldade de interação entre o átomo pesado e o analito. Alguns íons de átomos pesados tais como $\mathrm{Tl}^{+}$e I', também têm se mostrado mais sensíveis a esse efeito do que outros íons. Assim, soluções dos analitos foram depositadas em substratos de celulose, que por sua vez foram previamente tratadas com $5 \mu \mathrm{L}$ da solução de sal de átomo pesado apropriado (KI $\left.1,0 \mathrm{~mol} \mathrm{~L}^{-1}\right)$ e de $5 \mu \mathrm{L}$ de uma solução de SDS $0,07 \mathrm{~mol}$ $\mathrm{L}^{-1}$.

Além da preocupação com a obtenção dos parâmetros experimentais, uma eficiente detecção da fosforescência do(s) analito(s) também requer a otimização das variáveis instrumentais velocidade de varredura, tempo de retardo do detector, tempo de abertura do detector e abertura de 
fenda. Estes parâmetros são responsáveis pela resolução e magnitude do sinal do analito e do sinal de fundo obtido em cada medição.

A velocidade de varredura determina o tempo de integração da aquisição de dados. É sabido que a melhor razão sinal do analíto / sinal de fundo é obtida quando são escolhidas baixas velocidades. Além disso, a velocidade de varredura está relacionada com o aspecto e resolução do espectro observado. O tempo de abertura do detector determina o tempo de exposição do detector à radiação luminescente. O tempo de retardo, por sua vez, é um parâmetro fundamental em fosforimetria, já que permite rejeitar os fenômenos ópticos de curta duração (fluorescência e espalhamento) minimizando assim o ruído associado à medição de fosforescência.

Os parâmetros instrumentais temporais (tempo de abertura do detetor e tempo de retardo) foram também otimizados. O tempo de purga antes da medição do sinal, a velocidade de varredura e a abertura das fendas dos monocromadores foram fixadas de acordo com estudos anteriores [3, 6]. Esses resultados indicaram condições experimentais que permitiram o incremento do sinal líquido fosforescente e a aquisição dos espectros de fosforescência total.

Pode-se verificar que na ausência de íons de átomos pesados não existe sinal fosforescente proveniente dos componentes das cervejas em estudo. Nos espectros obtidos com a varredura de fosforescência total (Figuras 2 e 3) a região onde ocorre o espalhamento da radiação de excitação, que não é de interesse por ser bastante poluída em termos de sinal, foi evitada e a região de interesse (região de "impressão digital") que ocorre entre 210350/350-650 nm tomada para avaliação visual e estatística.

Os experimentos de varredura total indicaram sutis diferenças na composição dos produtos testados. Estas diferenças, seguramente relacionadas aos distintos processos de fabricação destas cervejas (variações no amargor, extrato, matérias-primas empregadas e tipo de fermentação) mostram, portanto, o potencial da aplicação da fosforimetria em substrato sólido na temperatura ambiente (FSSTA) para a classificação, caracterização e discriminação de cervejas. 
Revista Eletrônica TECCEN - ano I - volume 2 - Out-Dez de 2008 ISSN 1984-0993

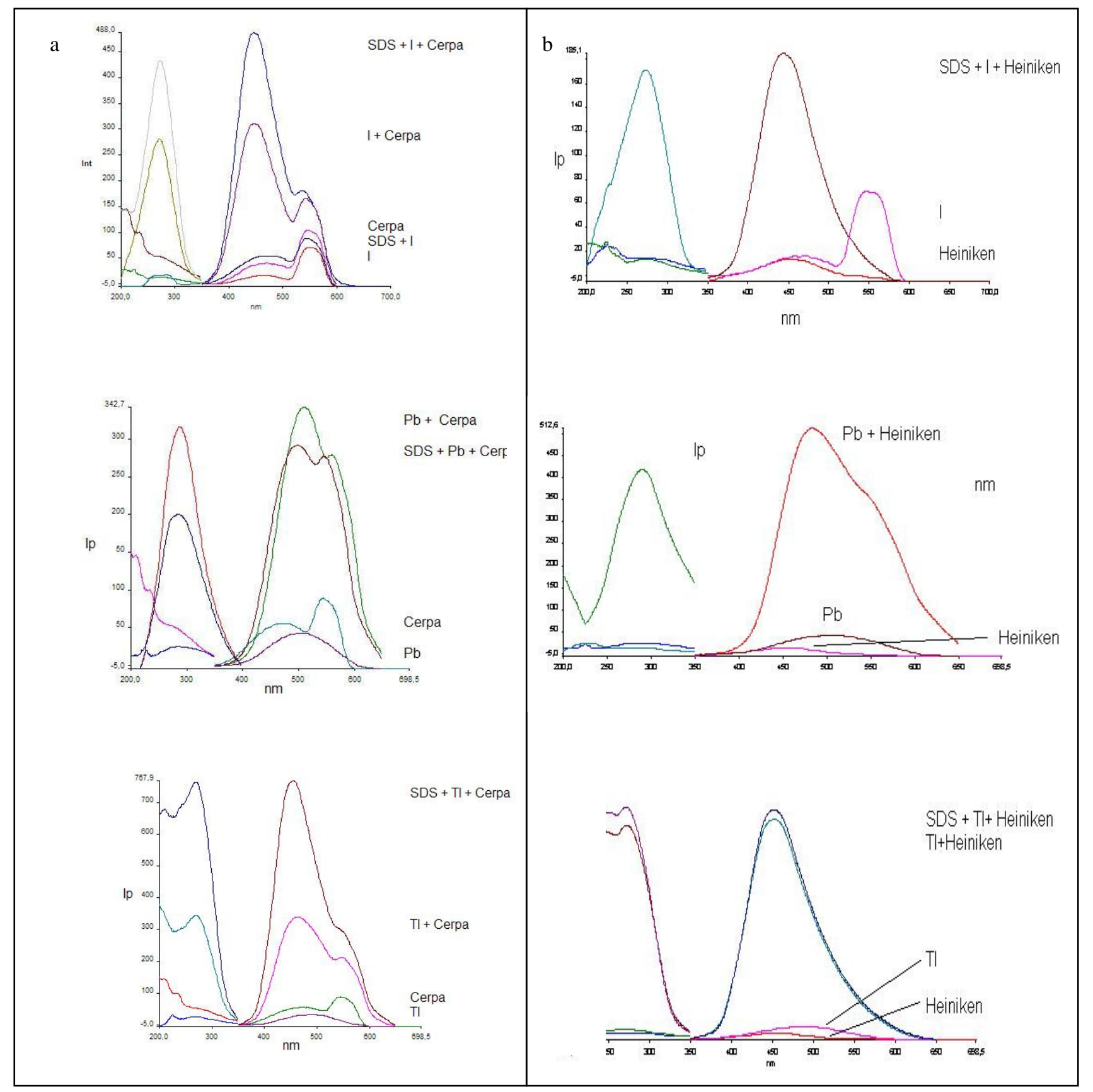

Figura 1: Espectros fosforescentes da cerveja Cerpa (a) e Heineken (b) em diferentes condições. 
Revista Eletrônica TECCEN - ano I - volume 2 - Out-Dez de 2008 ISSN 1984-0993

3D Cerpa
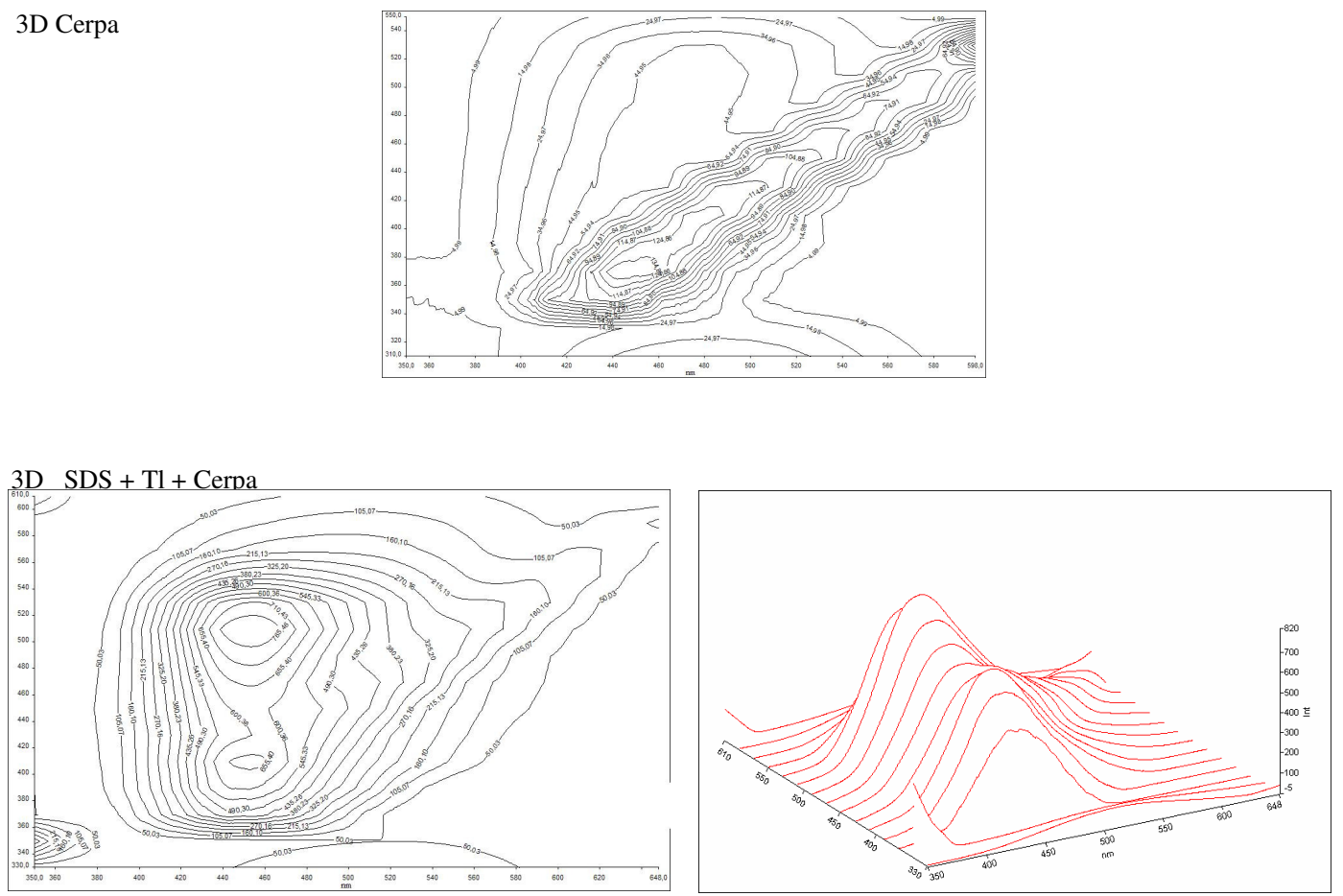

3D SDS $+\mathrm{I}+$ Cerpa
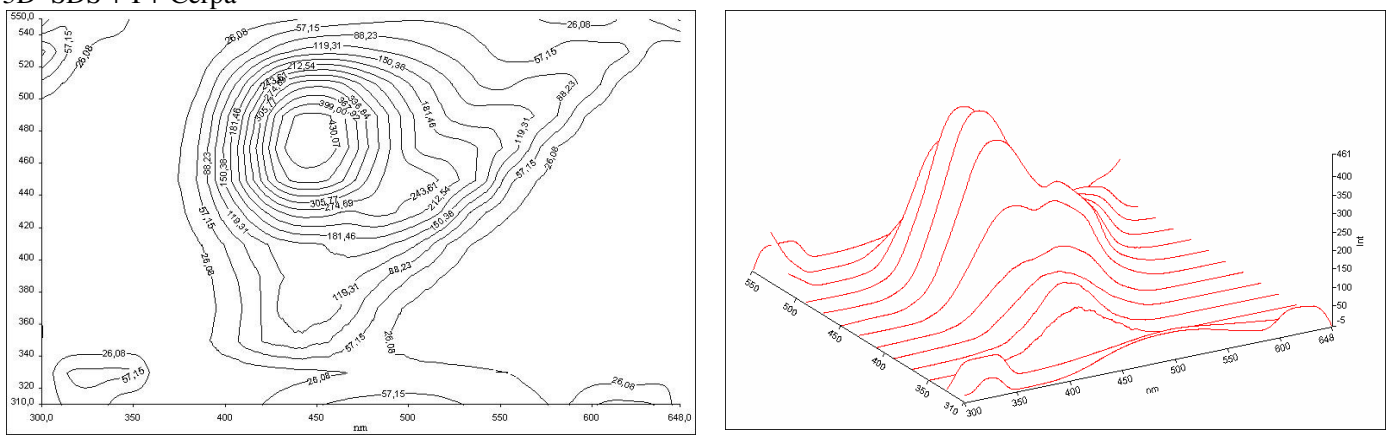

$3 \mathrm{D} \mathrm{Pb}+\mathrm{Cerpa}$
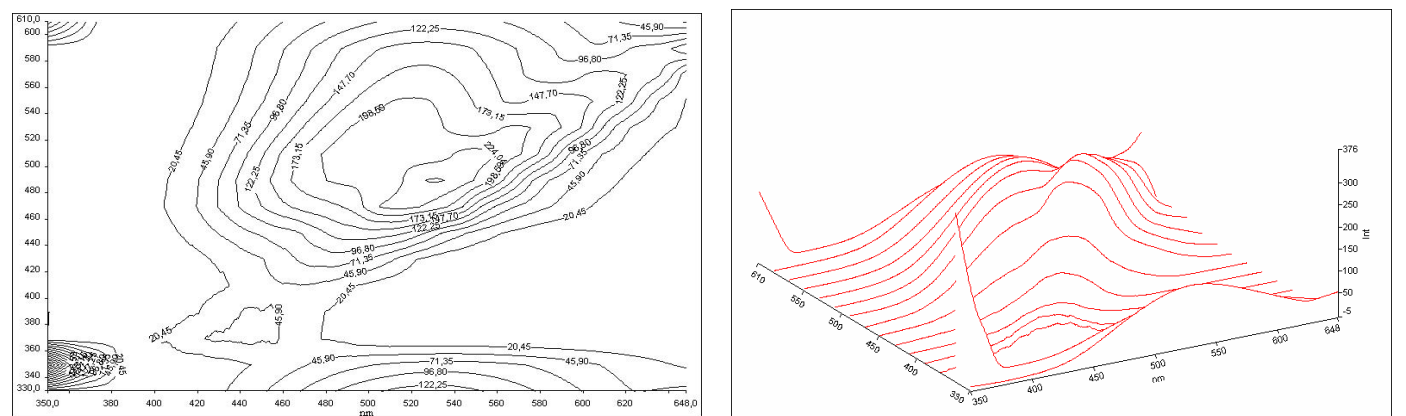

Figura 2: Mapas de contorno e superfícies de projeção da cerveja Cerpa. 
Revista Eletrônica TECCEN - ano I - volume 2 - Out-Dez de 2008 ISSN 1984-0993

3D Heineken

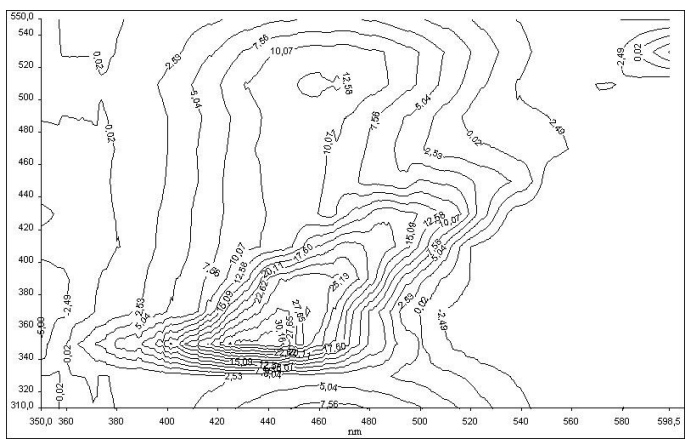

3D SDS $+\mathrm{Tl}+$ Heineken
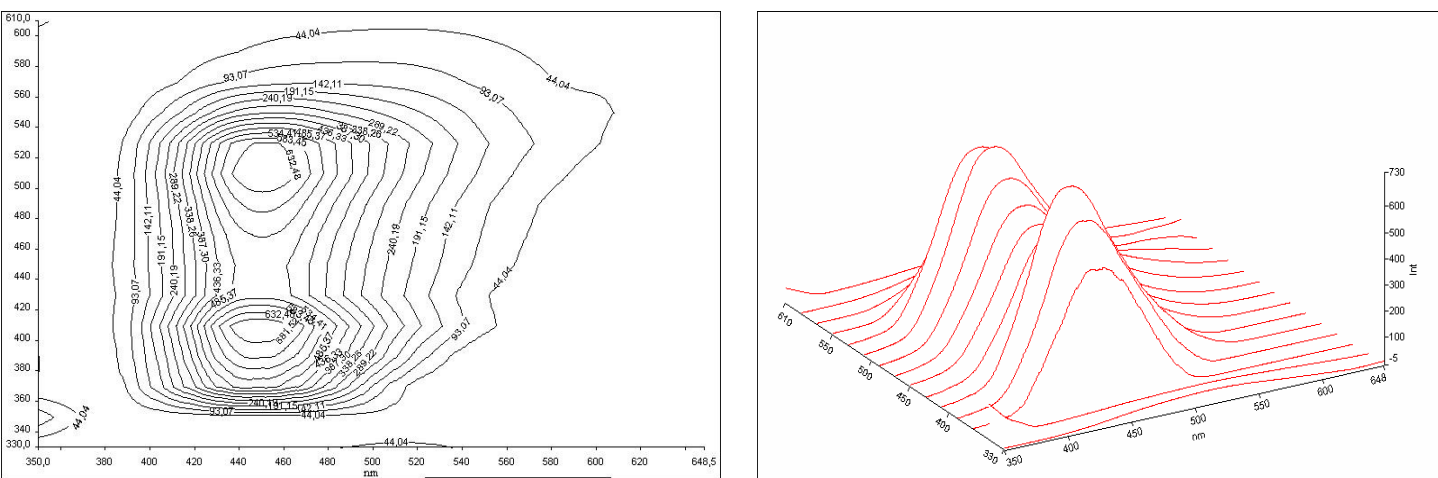

3D SDS+I + Heineken
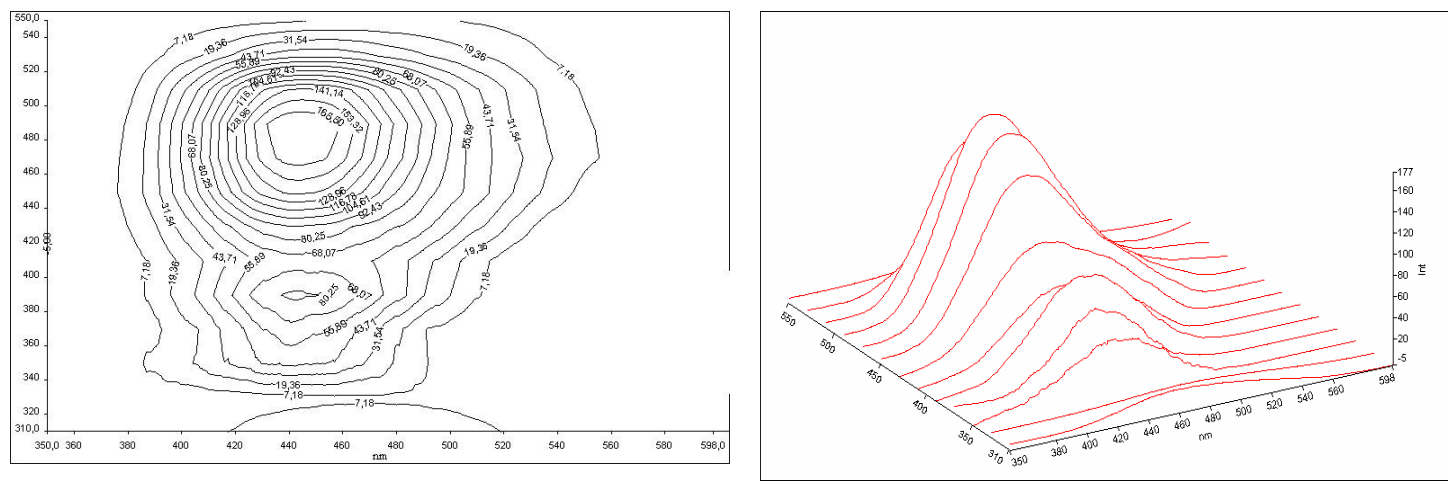

$3 \mathrm{D} \mathrm{Pb}+$ Heineken
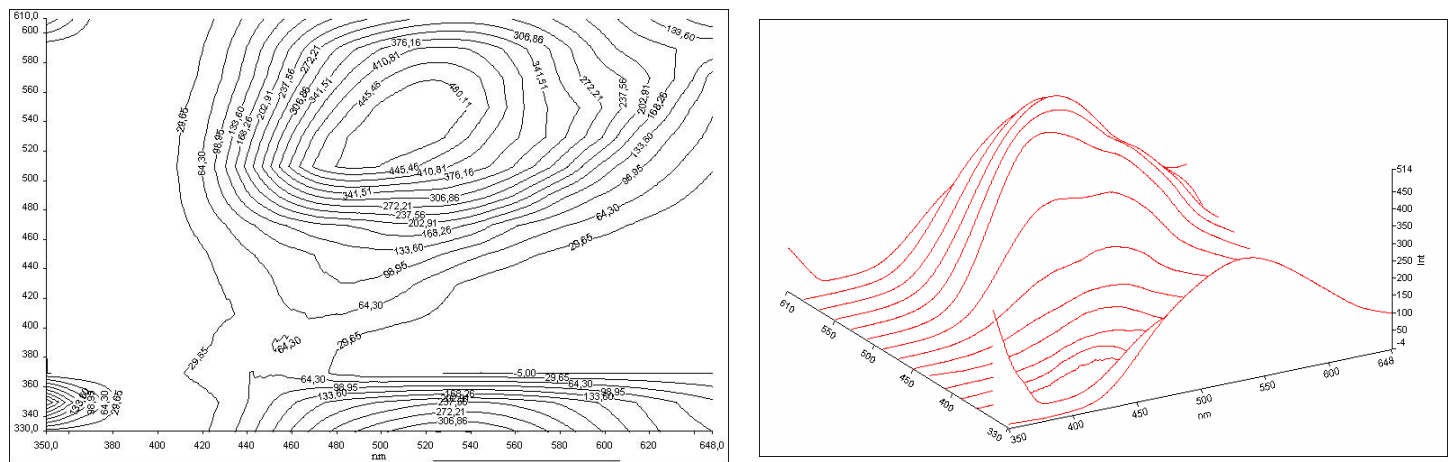

Figura 3: Mapas de contorno e superfícies de projeção da cerveja Heineken. 


\section{Conclusões}

Uma metodologia para caracterização de amostras de cervejas foi desenvolvida. Parâmetros experimentais e instrumentais foram otimizados para a indução e a maximização da razão sinal/sinal do branco. Condições experimentais foram estudadas e otimizadas com o intuito de minimizar a interferência entre os processos radiativos (fluorescência e fosforescência). Isso foi conseguido pela combinação do uso de surfactante SDS $0,07 \mathrm{~mol} \mathrm{~L}^{-1}$ e de átomo pesado, KI 1,0 mol L ${ }^{-1}$ e também pela utilização de tempos adequados de abertura do detector e tempo de retardo.

É importante salientar que devido à complexa composição da cerveja (aminoàcidos, vitaminas, proteínas de baixo, médio e alto peso molecular, entre outros), não se descarta a possibilidade de que outras substâncias presentes na amostra possam emitir sinal fosforescente similares aos observados. Desta forma, apesar dos resultados iniciais mostrarem-se bastante encorajadores, trabalhos futuros devem incluir a comparação dos resultados iniciais deste estudo com aqueles relacionados a padrões certificados e rastreáveis, além de incluírem um maior número de amostras.

\section{Referências}

[1] E. SIKORSKA et al. Food Chemistry, 96: 632639. 2006.

[2] D. BELLIDO-MILLA et al., Spectrochimica Acta Part B, 55: 855-864, 2000.

[3] B. CAÑABATE-DIAZ et al., Analyst, 128: 411415, 2003.

[4] A. D. CAMPÍGliA, C. G. DE LIMA, Anal. Chem., 59: 28-22, 1987.

[5] T. VO-DINH, Room Temperature Phosphorimetry for Chemical Analysis, Chemical analysis series v. 68, $1^{\text {st }}$ Ed., New York: .Jonh Wiley \& Sons, 1994.

[6] C. E. CARDOSO, R. Q. AUCÉLIO, Determination of benzo[b]naphtho[2,3-d]thiophene (BNT) in sediments using selective heavy atom induced room-temperature phosphorimetry. Journal of the Brazilian Chemical Society, 17(7): 13231328, 2006. 